\title{
Spatial Modulation of Nonlinear Flexural Vibrations of Membrane Resonators
}

\author{
Fan Yang, Felix Rochau, Jana S. Huber, Alexandre Brieussel, Gianluca Rastelli, Eva M. Weig, and Elke Scheer \\ Department of Physics, University of Konstanz, 78457 Konstanz, Germany
}

(Received 6 February 2019; revised manuscript received 13 March 2019; published 18 April 2019)

\begin{abstract}
We study the vibrational motion of mechanical resonators under strong drive in the strongly nonlinear regime. By imaging the vibrational state of rectangular silicon nitride membrane resonators and by analyzing the frequency response using optical interferometry, we show that, upon increasing the driving strength, the membrane adopts a peculiar deflection pattern formed by concentric rings superimposed onto the drum head shape of the fundamental mode. Such a circular symmetry cannot be described as a superposition of a small number of excited linear eigenmodes. Furthermore, different parts of the membrane vibrate at different multiples of the drive frequency, an observation that we denominate as "localization of overtones." We introduce a phenomenological model that is based on the coupling of a small number of effective nonlinear resonators, representing the different parts of the membrane, and that describes the experimental observations correctly.
\end{abstract}

DOI: 10.1103/PhysRevLett.122.154301

Micro- and nanoscale mechanical resonators bear rich potential to expand their applications in a broad range of areas, such as noise sensing [1], optomechanical (quantum) metrology [2,3], nanoelectromechanical logic gates [4,5], and parametric oscillators [6,7]. Mechanical resonators can have different size scales, and may be even as small as single carbon nanotubes or patterned graphene sheets $[8,9]$. Among larger devices, quasi-two-dimensional membrane resonators are interesting for several reasons, e.g., they have resonant behavior in a broad range of frequencies (e.g., from $100 \mathrm{kHz}$ to several $\mathrm{MHz}$ ) which make them attractive as broadband transducers for vibratory energy harvesting [10]. They are also important in hybrid engineered systems, since flexural modes can be easily coupled to other degrees of freedom such as photons [11-14] and cold atoms in hybrid optomechanical architectures $[15,16]$. Suspended membrane resonators operating in the singlemode regime have been studied in the weakly nonlinear limit and modeled as a Duffing resonator [17]. Increasing the membrane deflection amplitudes enforces the nonlinearity, thereby giving rise to nonlinear coupling between eigenmodes [18-20]. So far, experiments reported nonlinear interaction involving only two interacting modes [21-25], and only when matching an internal resonant condition between eigenfrequencies [26].

In this Letter, we analyze a novel, nonlinear vibrational state of a membrane resonator, achieved under strong drive. We observe the appearance of spatially modulated overtones. Upon increasing the driving strength, the membrane adopts a characteristic ring-shaped deflection pattern in which different parts of the membrane vibrate at different subharmonically excited multiples of the driving frequency. Such a ring-shaped pattern is distinctly different from the waveform and the symmetry of the flexural eigenmodes of the rectangular membrane. In other words, this regular pattern can only be reconstructed by using a superposition of a multitude of eigenmodes of the membrane. Moreover, the presence of subharmonically excited tones points out that the system is in a strongly nonlinear vibrational regime. Part of this phenomenon was reported before [27], but remained unexplained. Here we analyze it in detail and present a phenomenological model that captures the main features and semiquantitatively describes the experimental findings. The starting point of this model is that, under driving, the deflection profile of the membrane is well described by

$$
u(r, t) \simeq \sum_{n \geq 1} q_{n}(t) h_{n}(r) e^{i 2 n \pi f_{d} t}+\text { c.c. },
$$

where $f_{d}$ is the driving frequency and $r$ is the radial coordinate of the 2D membrane, $h_{n}(r)$ are arbitrary spatial profile functions, and the amplitudes of vibration are $q_{n}(t)$, respectively. The amplitudes $q_{n}(t)$ play the role of effective resonators with different eigenfrequencies $f_{n}$ and having sizeable amplitudes at different positions on the membrane, described by the functions $h_{n}(r)$, i.e., the localized overtones. We assume a nonlinear interaction $V=\sum_{n \geq 2} \lambda_{n} q_{1}^{n} q_{n}$ between a mode $q_{1}$ with eigenfrequency $f_{1}$ and its overtones. This model is referred to as a $1: n$ nonlinear coupling model.

We will show that, to describe our experimental observations, a small number of modes is sufficient, representing a significant simplification compared to the classical model based on linear eigenmodes of a rectangular membrane. Our conclusions are thus that spatially modulated overtones represent, indeed, a new paradigm in the nonlinear dynamics of membranes, which goes beyond previous theoretical 
pictures, based on the approach of nonlinear interaction of few linear eigenmodes [23-25].

The sample fabrication and measurement principles of the silicon nitride ( $\mathrm{SiN}$ ) membranes have been described in detail elsewhere [28-32] and are summarized in the Supplementary Material [33]. Flexural modes of the membrane are excited by applying an AC voltage $V_{\text {exc }} \sin \left(2 \pi f_{d} t\right)$ to a piezoelectric ring that causes a uniform thickness change of the piezoelectric ring actuator, resulting in an inertial excitation of the membrane, see Fig. 1(a). The vibrational state of the membrane in vacuum is

(a)

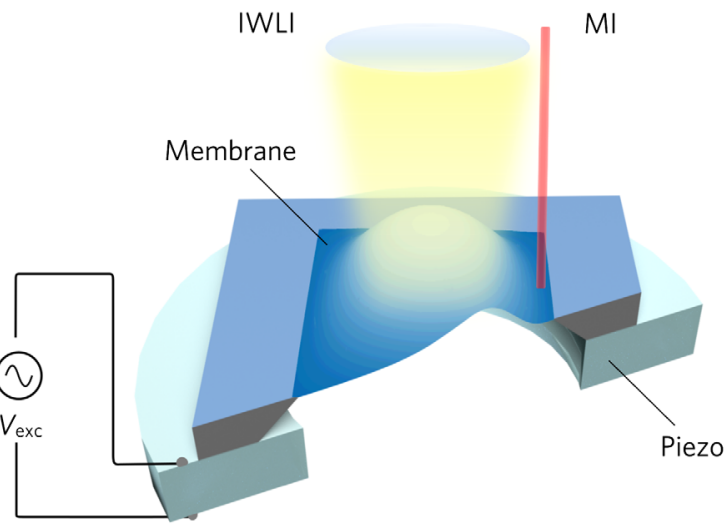

(b)

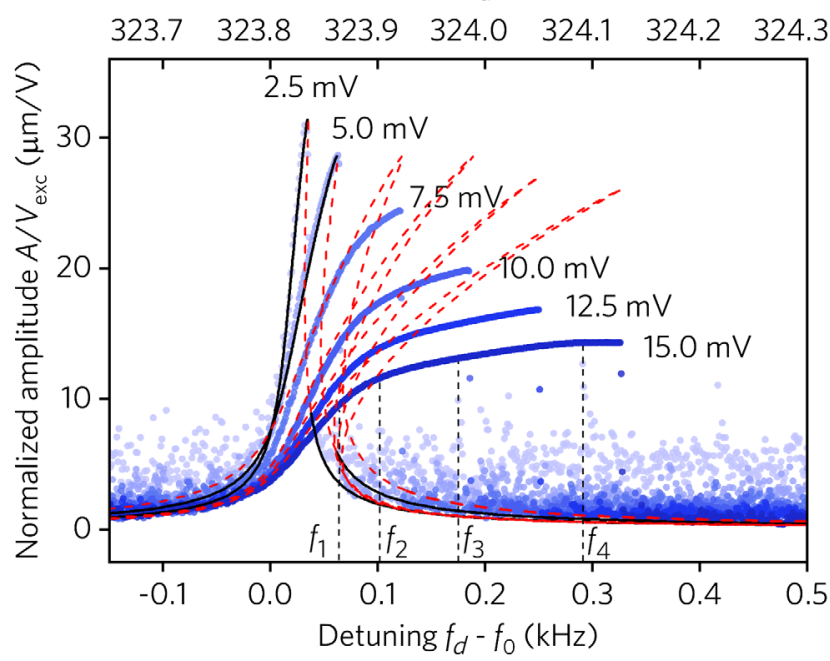

FIG. 1. (a) Sketch of the experimental setups showing the membrane chip carrying a free-standing $\mathrm{SiN}$ membrane, the piezoelectric ring and drive, the objective of the imaging white light interferometer (IWLI) and the laser beam of the Michelson interferometer (MI). (b) Nonlinear resonance curves (measured by IWLI and integrated over the entire membrane area) with varying $V_{\text {exc }}$, normalized to $V_{\text {exc }}$, are plotted vs the detuning frequency with respect to the linear eigenfrequency $f_{d}-f_{0}$. Here $f_{0}$ is the eigenfrequency of the fundamental mode $f_{0}=f_{(1,1)}$. The top axis shows the absolute frequency values measured with the IWLI. The red dashed lines are fits with the Duffing model. observed in two different set-ups providing two different types of optical interferometry. The imaging white light interferometer (IWLI) is able to spatially resolve the deflection profile and to obtain the average amplitude response by integrating the deflection profile over a selected area on the membrane surface [28]. The Michelson interferometer (MI) is focusing on one particular position of the membrane with a spot diameter of $\sim 2 \mu \mathrm{m}$. In this Letter, we show data recorded on the same membrane. Similar phenomena have been observed also for membranes with different size or made from different materials. An example is shown in the Supplemental Material [33]. Further experimental details are also given in the Supplemental Material [33]. In the linear response regime, we find resonances corresponding to the eigenfrequencies $f_{(m, n)}$ of the flexural eigenmodes which are characterized by the integers $m$ and $n$ indicating the number of deflection maxima in the two spatial directions of the membrane plane [32]. For example, we find $f_{(1,1)}=$ $321 \mathrm{kHz}$ and $f_{(2,2)}=646 \mathrm{kHz}$ for the eigenfrequencies of the $(1,1)$ and $(2,2)$ mode, respectively, both with quality factors in the order of 20000 , see the Supplemental Material [33] for more details. The eigenfrequencies of all modes discussed in this article as well as further mechanical parameters are listed in Table 1 of the Supplemental Material. We utilize the IWLI signal integrated over the entire membrane area to record the nonlinear vibration behavior under intermediate and strong sinusoidal excitation and with a driving frequency $f_{d}$ around $f_{(1,1)}$. With $V_{\text {exc }}=2.5 \mathrm{mV}$ to $5.0 \mathrm{mV}$, the resonance curves exhibit a Duffing-type nonlinearity, as shown in Fig. 1(b), but when exceeding $V_{\text {exc }}>5.0 \mathrm{mV}$, the amplitude rises more weakly than expected for the Duffing model. This observation signals the onset of the spatial modulation phenomenon. The spatial deflection profiles captured by IWLI for $V_{\text {exc }}=15.0 \mathrm{mV}$ are shown in Fig. 2(a). When continuously sweeping the driving frequency $f_{d}$, the spatial appearance of the deflection pattern changes from single drum head type at $f_{d}=f_{1}$ [see marker in Fig. 1(b)] to a crater type at $f_{d}=f_{2}$, where the amplitude at the membrane center decreases and the central part of the deflection pattern adopts a flat shape.

When further increasing $f_{d}$ to $f_{3}$, the flat area evolves into a separate drum head shape. Finally, at $f_{4}$ again a minimum occurs in the center surrounded by two rings. The observed deflection patterns reveal a frequency-dependent spatial modulation of the $(1,1)$ mode, which cannot be described as a simple superposition of few $(m, n)$ modes, since it has a circular symmetry contrary to the axial symmetry of the higher $(m, n)$ modes in a rectangular membrane. With the help of sweep-up and ring-down experiments performed in the MI at different positions on the membrane, one can reveal that the modulation is caused by frequencies that are identified as overtones of 

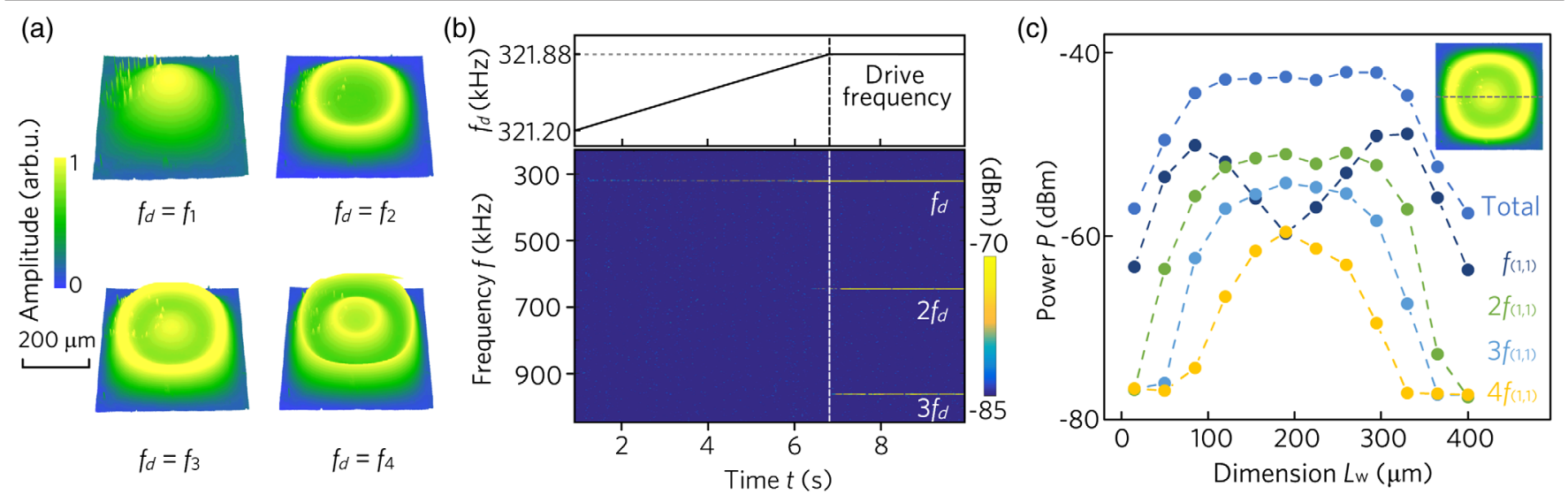

FIG. 2. Localized overtones for near-resonant drive in the spatial modulation regime. (a) Spatial deflection patterns observed at different driving frequencies $f_{d}$ denoted as $f_{1}, f_{2}, f_{3}$, and $f_{4}$ in Fig. 1(b), associated with the spatial overtones of the (1,1) mode $\left(V_{\text {exc }}=15 \mathrm{mV}\right)$. (b) Power spectrum (measured by MI) of a sweep-up measurement around the $(1,1)$ mode up to $f_{d}=321.88 \mathrm{kHz}$ (dashed line), from where $f_{d}$ is kept constant, $V_{\mathrm{exc}}=50 \mathrm{mV}$. Top: drive frequency as a function of time. (c) Position dependence of relative amplitude of overtones recorded at $V_{\mathrm{exc}}=35 \mathrm{mV}$ and $f_{d} \approx f_{3}$ [measured by $\mathrm{MI}$ ] for a line cut parallel to the edges through the center of the membrane, see schematic in the inset and Fig. 2(a). The intensities of the individual overtones (up to fourth overtone shown) and the total intensity are obtained from the sweep-up measurements.

the $(1,1)$ mode with $2 f_{(1,1)}$ and $3 f_{(1,1)}$, as we will show in the following.

We perform a slow sweep-up of $f_{d}$ at constant $V_{\text {exc }}$ from a starting value below the resonance frequency of a particular mode up to a maximum frequency above the resonance frequency but within the frequency range of the high response amplitude. At this frequency we switch off the drive. The measurement spot is close to one edge of the membrane and chosen such that the maximum deflection stays smaller than a quarter of the laser wavelength (see Supplemental Material [33] for details). Note that due to experimental constraints the excitations and axis scalings in Figs. 2(a)-2(c) are different. A direct comparison between the mode shapes $n$ Fig. 2(a) and the amplitudes in Fig. 2(c) is therefore not possible. A fast Fourier transform (FFT) algorithm is performed on the time domain deflection displacement data in narrow time windows to get a time-resolved power spectrum of the sweep-up and ring-down processes. Figure 2(b) shows the time evolution during the sweep-up of the power spectrum for a moderate excitation from a starting frequency slightly above the linear eigenfrequency $f_{0}$ up to $f_{d}=321.88 \mathrm{kHz}$. The subsequent ring-down part is displayed in Fig. S10 in the Supplemental Material [33]. In contrast to the linear response regime shown in Fig. S9 in the Supplemental Material, we observe response at multiple frequencies which are setting in one after another at different $f_{d}$. Strong signals are observed at $f_{d}, 2 f_{d}$, and $3 f_{d}$, respectively. These responses evolve during the ring-down process towards the overtones of the first mode, namely to $f_{(1,1)}, 2 f_{(1,1)}$, and $3 f_{(1,1)}$. We note that $2 f_{(1,1)}=642 \mathrm{kHz}$ can clearly be distinguished from the eigenfrequency $f_{(2,2)}=646 \mathrm{kHz}$ of the $(2,2)$ mode, given the frequency resolution of the FFT algorithm $\sim 1 \mathrm{kHz}$, which is larger than the intrinsic line width of the linear resonances of $\sim 50 \mathrm{~Hz}$. A similar multiple frequency response has also been found for $\mathrm{SiC}$ and Ge membrane systems [27]. The multiple frequency response complements the observations from the IWLI and gives a strong hint that the spatial modulation state corresponds to spatially varying superpositions of the fundamental mode and its overtones. In addition, stroboscopic IWLI measurements show [see Fig. S6(d) in the Supplemental Material], that at $f_{d}=f_{3}$ the central part of the membrane (inner disk with a drum head shape) vibrates at a different frequency than the outer annulus which moves at $f_{d}$.

To further test this hypothesis of localized overtones we perform MI measurements at different positions on the membrane. Figure 2(c) shows the relative amplitudes of the overtones observed in the power spectrum for a line profile from one edge of the membrane to the other, crossing the center of the membrane, see the inset of Fig. 2(c). At each spot a sweep-up is performed and the intensities of the overtones are evaluated at fixed detuning by separately integrating the power of each time window within a corresponding frequency range. The total intensity reveals a large plateau that extends over a width of roughly $200 \mu \mathrm{m}$ in the center of the membrane. The driving frequency $f_{d} \simeq$ $f_{(1,1)}$ has the highest amplitude at the outer rim of this plateau and strongly decreases towards the center. In the central part the intensity of the second overtone exceeds the one of the ground tone. The third and fourth multiples of $f_{d}$ have a weaker intensity and are even more centered. This observation evidences the existence of spatially localized overtones, confirming the interpretation of the IWLI data. 
The detailed microscopic analysis of the observed spatial overtones and their appearance is a challenging theoretical task and beyond the aim of this Letter. The main physical aspects can be captured by the phenomenological ansatz given in Eq. (1) that describes that the membrane is vibrating at distinct sections at different frequencies. We note that any profile function can be obtained as a linear combination of the eigenmodes $(m, n)$ since the latter form a complete orthonormal basis. An example is given in the Supplemental Material [33] in which we project the ring shape of the deflection onto the linear eigenmodes. This yields a very slowly convergent series: as expected intuitively, a superposition of many modes is needed to reproduce a circular ring shape $(N \geq 100)$ of the rectangular membrane (see Supplemental Material [33]). By contrast, the spatially modulated overtones $u_{n}$ can be viewed as renormalized eigenmodes appearing only for strong driving. They can be mathematically defined on the concept of an invariant manifold in an elastic nonlinear medium [34]. For simplicity we describe here the situation with only two resonators, i.e., the 1:2 model. The general $1: n$ model is detailed in the Supplemental Material [33] and in Refs. [26,35]. We thus consider the equation

$u(r, t) \simeq q_{2}(t) h_{2}(r) e^{i 4 \pi f_{d} t}+q_{1}(t) h_{1}(r) e^{i 2 \pi f_{d} t}+$ c.c.,

where $h_{1}(r)$ is localized at a ring of radius $R$ from the center and has the eigenfrequency $f_{1} \simeq f_{(1,1)}$ and $h_{2}(r)$, is localized at the center, with eigenfrequency $2 f_{1}$, as shown in Fig. 2(a) for $f_{d}=f_{3}$.

Finally, in our model, we assume a nonlinear coupling between the two effective resonators, and we set the following coupled equations for the amplitudes $q_{1}$ and $q_{2}$ :

$$
\begin{aligned}
& \ddot{q}_{1}(t)=-\left(2 \pi f_{1}\right)^{2} q_{1}(t)-2 \Gamma_{1} \dot{q}_{1}(t)-2 \lambda q_{1}(t) q_{2}(t) \\
&-\gamma_{1} q_{1}^{3}(t)+F_{\mathrm{d}} \cos \left(2 \pi f_{d} t\right), \\
& \ddot{q}_{2}(t)=-4\left(2 \pi f_{1}\right)^{2} q_{2}(t)-2 \Gamma_{2} \dot{q}_{2}(t)-\lambda q_{1}^{2}(t) .
\end{aligned}
$$

Equations (3) and (4) mean that the membrane is effectively composed of an inner disc coupled to an outer ring [36]. The ring resonator is characterized by a Duffing nonlinearity with strength $\gamma_{1}$, and both are damped with damping constants $\Gamma_{1}$ and $\Gamma_{2}$. Most importantly, we also postulate a nonlinear resonant interaction between the two resonators with the interaction strength $\lambda$, which can be derived from the nonlinear potential $V=\lambda q_{1}{ }^{2} q_{2}$. This interaction is important since it implies a correlated behavior of the two amplitudes that we actually observe in the system, as we discuss next.

In Fig. 3 we show more curves from the same data set as depicted in Fig. 1(b). The orange lines are the same fits to the Duffing model as in Fig. 1(b), the green curves are calculated with the $1: 2$ coupling model and the blue curves

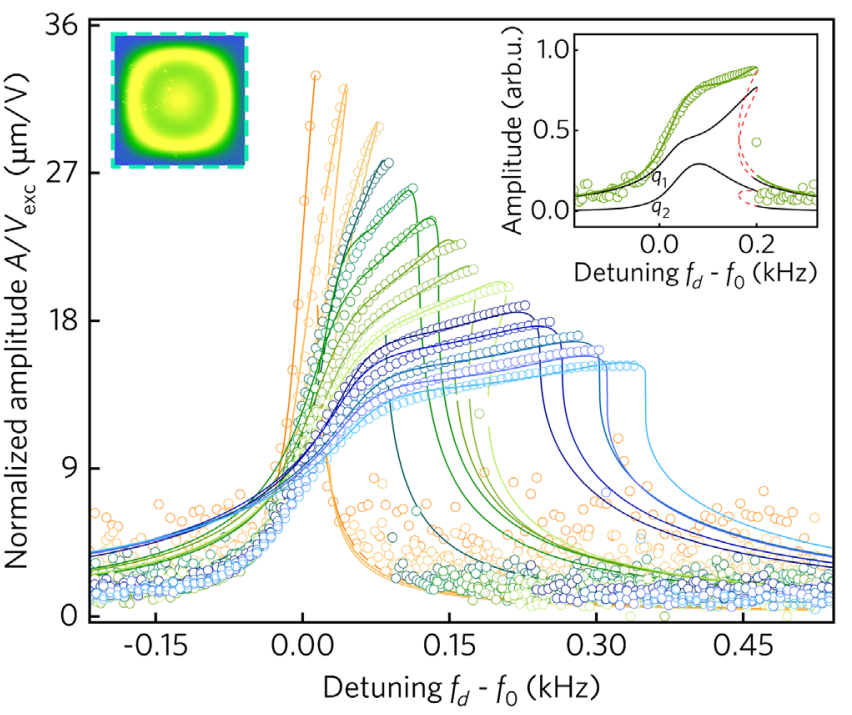

FIG. 3. Experimental responses (symbols) integrated over the entire membrane (shown as green frame in the inset) measured by IWLI for $V_{\text {exc }}=2.5,4.2,5.8,6.7,7.5,8.3,9.1,10.0,10.8,11.6$, $12.5,13.3,14.1$, and $15.0 \mathrm{mV}$. The lines are fits with either the Duffing or the coupled model. For details see text. Inset: Fitting of the curve recorded at $10.0 \mathrm{mV}$. The black lines show the individual contributions of modes $n=1$ and $n=2$.

using $1: 2$ and $1: 3$ coupling. The fitting parameters and their analyses are given in the Supplemental Material [33]. The model fits the experimental curves well. The small deviations can be explained by the simplicity of the model, e.g., neglecting the contributions of the higher overtones.

The analysis of the development of the spatial overtones is completed by studying the deflection amplitude in a small area. Figure 4(a) shows examples of response curves integrated over a square with size $80 \times 80 \mu \mathrm{m}^{2}$ in the central area [see dashed frame in inset of Fig. 4(b)] of the membrane for increasing $V_{\text {exc }}$. A zoom into the maxima is given in panel (b). It reveals a series of maxima and minima, before the amplitude eventually breaks down. The number of observable maxima increases with $V_{\text {exc }}$ in agreement with the increasing plateau length when integrating over the whole membrane area. For small $V_{\text {exc }}=$ $10 \mathrm{mV}$ only the first maximum and minimum are accessible, for $V_{\text {exc }}=93 \mathrm{mV}$ four maxima and minima are obtained. We interpret this observation as follows: upon increasing $f_{d}$, the diameter of the circular shaped overtones increase. The first maximum signals that $u_{1}$ reaches the edges of the integration window, thereby reducing the averaged amplitude. When further increasing $f_{d}$, more overtones are excited, increasing the amplitude and subsequently leaving the central area, thereby reducing the averaged amplitude again. As an example we fit the curve recorded for $V_{\text {exc }}=10 \mathrm{mV}$ with our theoretical model, comprising two tones, $q_{1}$ and $q_{2}$. To account for the fact that upon increasing $f_{d}$, the radius of the ring increases and partially leaves the integration area, we use a linearly 


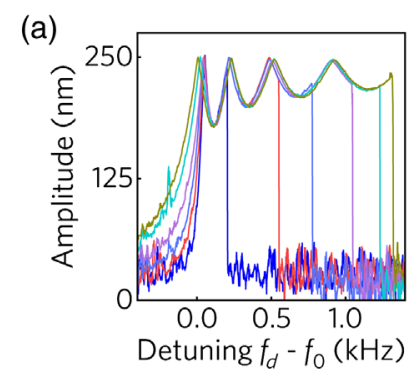

(c)

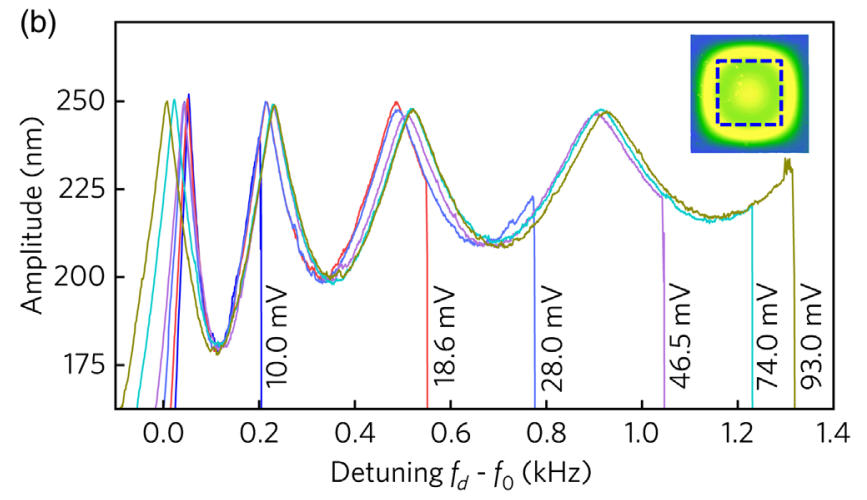

FIG. 4. Resonance curves measured in a localized area of the membrane. (a) Normalized averaged amplitude responses of the central area of the membrane (blue frame in inset deflection pattern), measured by IWLI curve for different $V_{\text {exc }}$ from $10 \mathrm{mV}$ up to $93 \mathrm{mV}$. (b) Zoom into the maximum region. (c) Experimental curve (symbols) for $V_{\text {exc }}$ from $10 \mathrm{mV}$ and fit (line) to the $1: 2$ model.

decreasing weight factor for mode $q_{1}$, see Supplemental Material [33]. The solid line in Fig. 4(c) is the result of the fitting with the theoretical model which describes the experimental observation qualitatively correctly. The individual contributions and the total fitted amplitude and further details are given in the Supplemental Material [33]. This concept can straightforwardly be extended to the situation with three and more modes. However, the number of fitting parameters increases rapidly. Summarizing, the deflection amplitude in the central area of the membrane can be interpreted as the gradual transfer of vibrational energy from $q_{1}$ to higher overtones $q_{n}$.

In conclusion, by combination of two complementary experimental approaches and the nonlinear $1: n$ coupling model we revealed a novel deflection state caused by a nonlinear coupling mechanism between several overtones of a single flexural mode of a membrane resonator. This deflection state is hallmarked by a spatial modulation pattern, in which different parts of the membrane vibrate at different frequencies. For rectangular membranes the spatial modulation state can be distinguished from the linear deflection pattern by a distinctly different symmetry. The coupling mechanism is mediated by nonlinear interaction and activated by strong driving.

The authors thank R. Waitz for help in sample fabrication. We are indebted to W. Belzig, T. Dekorsy, M. Dykman,
V. Gusev, M. Hettich, P. Leiderer, S. Shaw, I. Wilson-Rae, R. S. Edwards, L. Q. Zhou, and the SFB767 Nanomechanics Discussion Group for fruitful discussion and comments about the work. The authors gratefully acknowledge financial support from the China Scholarship Council, the European Union's Horizon 2020 program for Research and Innovation under Grant Agreement No. 732894 (FET Proactive HOT), by the German Excellence Initiative through the Zukunftskolleg of the University of Konstanz, and by the Deutsche Forschungsgemeinschaft (DFG) via the Collaborative Research Center SFB767.

*elke.scheer@uni-konstanz.de

[1] P. Weber, J. Güttinger, A. Noury, J. Vergara-Cruz, and A. Bachtold, Force sensitivity of multilayer graphene optomechanical devices, Nat. Commun. 7, 12496 (2016).

[2] N. S. Kampel, R. W. Peterson, R. Fischer, P.-L. Yu, K. Cicak, R. W. Simmonds, K. W. Lehnert, and C. A. Regal, Improving Broadband Displacement Detection with Quantum Correlations, Phys. Rev. X 7, 021008 (2017).

[3] M. Aspelmeyer, T. J. Kippenberg, and F. Marquardt, Cavity optomechanics, Rev. Mod. Phys. 86, 1391 (2014).

[4] I. Mahboob and H. Yamaguchi, Bit storage and bit flip operations in an electromechanical oscillator, Nat. Nanotechnol. 3, 275 (2008).

[5] D. N. Guerra, A. R. Bulsara, W. L. Ditto, S. Sinha, K. Murali, and P. Mohanty, A noise-assisted reprogrammable nanomechanical logic gate, Nano Lett. 10, 1168 (2010).

[6] D. Rugar and P. Grütter, Mechanical Parametric Amplification and Thermomechanical Noise Squeezing, Phys. Rev. Lett. 67, 699 (1991).

[7] M. J. Seitner, M. Abdi, A. Ridolfo, M. J. Hartmann, and E. M. Weig, Parametric Oscillation, Frequency Mixing, and Injection locking of Strongly Coupled Nanomechanical Resonator Modes, Phys. Rev. Lett. 118, 254301 (2017).

[8] A. Eichler, J. Moser, J. Chaste, M. Zdrojek, I. Wilson-Rae, and A. Bachtold, Nonlinear damping in mechanical resonators made from carbon nanotubes and graphene, Nat. Nanotechnol. 6, 339 (2011).

[9] X. Zhang, R. Waitz, F. Yang, C. Lutz, P. Angelova, A. Gölzhäuser, and E. Scheer, Vibrational modes of ultrathin carbon nanomembrane mechanical resonators, Appl. Phys. Lett. 106, 063107 (2015).

[10] Y. Jia, S. Du, and A. A. Seshia, Twenty-eight orders of parametric resonance in a microelectromechanical device for multi-band vibration energy harvesting, Sci. Rep. 6, 30167 (2016).

[11] J. C. Sankey, C. Yang, B. M. Zwickl, A. M. Jayich, and J. G. Harris, Strong and tunable nonlinear optomechanical coupling in a low-loss system, Nat. Phys. 6, 707 (2010).

[12] T. P. Purdy, R. W. Peterson, and C. Regal, Observation of radiation pressure shot noise on a macroscopic object, Science 339, 801 (2013).

[13] R. W. Andrews, R. W. Peterson, T. P. Purdy, K. Cicak, R. W. Simmonds, C. A. Regal, and K. W. Lehnert, Bidirectional and efficient conversion between microwave and optical light, Nat. Phys. 10, 321 (2014). 
[14] H. Xu, D. Mason, L. Jiang, and J. Harris, Topological energy transfer in an optomechanical system with exceptional points, Nature (London) 537, 80 (2016).

[15] S. Camerer, M. Korppi, A. Jöckel, D. Hunger, T. W. Hänsch, and P. Treutlein, Realization of an Optomechanical Interface Between Ultracold Atoms and a Membrane, Phys. Rev. Lett. 107, 223001 (2011).

[16] A. Jöckel, A. Faber, T. Kampschulte, M. Korppi, M. T. Rakher, and P. Treutlein, Sympathetic cooling of a membrane oscillator in a hybrid mechanical-atomic system, Nat. Nanotechnol. 10, 55 (2015).

[17] T. Antoni, K. Makles, R. Braive, T. Briant, P.-F. Cohadon, I. Sagnes, I. Robert-Philip, and A. Heidmann, Nonlinear mechanics with suspended nanomembranes, Europhys. Lett. 100, 68005 (2012).

[18] A. H. Nayfeh and D. T. Mook, Nonlinear Oscillations (John Wiley \& Sons, New York, 2008).

[19] A. I. Manevich and L. I. Manevitch, The Mechanics of Nonlinear Systems with Internal Resonances (World Scientific, Singapore, 2005).

[20] H. G. Schuster, Reviews of Nonlinear Dynamics and Complexity (John Wiley \& Sons, New York, 2009).

[21] C. Van der Avoort, R. Van der Hout, J. Bontemps, P. Steeneken, K. Le Phan, R. Fey, J. Hulshof, and J. Van Beek, Amplitude saturation of MEMS resonators explained by autoparametric resonance, J. Micromech. Microeng. 20, 105012 (2010).

[22] H. J. R. Westra, M. Poot, H. S. J. Van der Zant, and W. J. Venstra, Nonlinear Modal Interactions in ClampedClamped Mechanical Resonators, Phys. Rev. Lett. 105, 117205 (2010).

[23] D. Antonio, D. H. Zanette, and D. López, Frequency stabilization in nonlinear micromechanical oscillators, Nat. Commun. 3, 806 (2012).

[24] J. Güttinger, A. Noury, P. Weber, A. M. Eriksson, C. Lagoin, J. Moser, C. Eichler, A. Wallraff, A. Isacsson, and A. Bachtold, Energy-dependent path of dissipation in nanomechanical resonators, Nat. Nanotechnol. 12, 631 (2017).

[25] C. Chen, D. H. Zanette, D. A. Czaplewski, S. Shaw, and D. López, Direct observation of coherent energy transfer in nonlinear micromechanical oscillators, Nat. Commun. 8, 15523 (2017).
[26] O. Shoshani, S. Shaw, and M. Dykman, Anomalous decay of nanomechanical modes going through nonlinear resonance, Sci. Rep. 7, 18091 (2017).

[27] L. Zhou, G. Colston, M. Pearce, R. Prince, M. Myronov, D. Leadley, O. Trushkevych, and R. Edwards, Non-linear vibrational response of $\mathrm{Ge}$ and $\mathrm{SiC}$ membranes, Appl. Phys. Lett. 111, 011904 (2017).

[28] S. Petitgrand, R. Yahiaoui, K. Danaie, A. Bosseboeuf, and J. Gilles, 3D measurement of micromechanical devices vibration mode shapes with a stroboscopic interferometric microscope, Optics and Lasers in Engineering 36, 77 (2001).

[29] C. M. Hernandez, T. W. Murray, and S. Krishnaswamy, Photoacoustic characterization of the mechanical properties of thin films, Appl. Phys. Lett. 80, 691 (2002).

[30] R. Waitz, S. Nößner, M. Hertkorn, O. Schecker, and E. Scheer, Mode shape and dispersion relation of bending waves in thin silicon membranes, Phys. Rev. B 85, 035324 (2012).

[31] R. Waitz, C. Lutz, S. Nößner, M. Hertkorn, and E. Scheer, Spatially Resolved Measurement of the Stress Tensor in Thin Membranes Using Bending Waves, Phys. Rev. Applied 3, 044002 (2015).

[32] F. Yang, R. Waitz, and E. Scheer, Quantitative determination of the mechanical properties of nanomembrane resonators by vibrometry in continuous light, arXiv:1704.05328.

[33] See Supplemental Material at http://link.aps.org/ supplemental/10.1103/PhysRevLett.122.154301 for details of the sample preparation and the measurement methods, characterization measurements of the membrane under study, examples of spatial modulation phenomena observed for higher flexural modes, the detailed description of the theoretical model, the fitting procedure and fit results as well as ring-down.

[34] E. Pesheck, N. Boivin, C. Pierre, and S. W. Shaw, Nonlinear modal analysis of structural systems using multi-mode invariant manifolds, Nonlinear Dyn. 25, 183 (2001).

[35] F. Mangussi and D. H. Zanette, Internal resonance in a vibrating beam: a zoo of nonlinear resonance peaks, PLoS One 11, e0162365 (2016).

[36] A. Bijari, S.-H. Keshmiri, and F. Babazadeh, Nonlinear modeling for distortion analysis in silicon bulk-mode ring resonators, Micromachines 3, 582 (2012). 